\title{
Application of Numerical Inverse Laplace Transform Methods for Simulation of Distributed Systems with Fractional-Order Elements*
}

\author{
Nawfal Al-Zubaidi R-Smith ${ }^{\dagger}$, Aslihan Kartci ${ }^{\ddagger}$ and Lubomír Brančík ${ }^{\S}$ \\ Department of Radio Electronics, Brno University of Technology, \\ Technicka 12, Brno, Czech Republic \\ ${ }^{\dagger}$ alzubaidi@phd.feec.vutbr.cz \\ \$kartci@feec.vutbr.cz \\ $\S$ brancik@feec.vutbr.cz
}

Received 26 October 2017

Accepted 7 December 2017

Published 15 January 2018

\begin{abstract}
The paper presents a computationally efficient method for modeling and simulating distributed systems with lossy transmission line (TL) including multiconductor ones, by a less conventional method. The method is devised based on 1D and 2D Laplace transforms, which facilitates the possibility of incorporating fractional-order elements and frequency-dependent parameters. This process is made possible due to the development of effective numerical inverse Laplace transforms (NILTs) of one and two variables, 1D NILT and 2D NILT. In the paper, it is shown that in high frequency operating systems, the frequency dependencies of the system ought to be included in the model. Additionally, it is shown that incorporating fractional-order elements in the modeling of the distributed parameter systems compensates for losses along the wires, provides higher degrees of flexibility for optimization and produces more accurate and authentic modelling of such systems. The simulations are performed in the Matlab environment and are effectively algorithmized.
\end{abstract}

Keywords: Distributed system; fractional calculus; frequency dependence; Laplace transform; numerical inversion; Matlab; transmission line.

\section{Introduction}

Numerical inverse Laplace transform (NILT) methods are ranked among the potential methods for the analysis of transient behavior of linear dynamical systems. The NILT methods differ from each other in their accuracy, computation

*This paper was recommended by Regional Editor Piero Malcovati.

$\dagger$ Corresponding author.

This is an Open Access article published by World Scientific Publishing Company. It is distributed under the terms of the Creative Commons Attribution 4.0 (CC-BY) License. Further distribution of this work is permitted, provided the original work is properly cited. 
effectiveness, the difficulty of optimizing the methods' parameters and the versatility of transforms and applications to cover. Several 1D NILT methods have been developed, though the methods based on FFT techniques prove to be convenient from a practical point of view, when the inversions are relatively fast and accurate; such as the FFT 1D NILT method. ${ }^{1,2}$ In Ref. 3, a 2D-NILT method based on the sum of infinite 2D complex Fourier series partially evaluated by FFT was presented, subsequently enhanced by adapting the $\epsilon$-algorithm of $\mathrm{Wynn}^{4}$ and the quotient-difference (qd) algorithm of Rutishauser. ${ }^{5}$ Besides, a method based on the approximation of the Laplace transform's inverse kernel, $\exp (s t)$, in the inverse Laplace transform (ILT) definition of Bromwich integral was developed and are at disposal. ${ }^{6,7}$ For this method, several convergence acceleration techniques were investigated and tested for its accuracy and computation speed; particularly the epsilon algorithm of Wynn, the qd algorithm of Rutishauser ${ }^{8}$ and the Euler transform. ${ }^{7}$ In this paper, the hyperbolic NILT method ${ }^{8}$ is used to get the 2D results, whereas, the FFT NILT method generalized to be able to invert Laplace transforms in matrix forms is used for the $3 \mathrm{D}$ results $^{5}$; both methods are accelerated via the qd algorithm. The second method benefits from the capabilities of the Matlab language to process multi-dimensional arrays and several mathematical operations in parallel. The advantage of this process is mainly to save valuable computational time and simplify the computation process.

Fractional calculus, the branch of mathematics regarding differentiations and integrations to noninteger orders, is a field that was introduced 300 years ago. ${ }^{9}$ Inspired by the fractal models in the environment, integer to noninteger models were explored. It originates from the correspondence between Leibniz and LHopital on 30th September, 1695, with LHopital inquiring about Leibnizs notation, $d^{n} y / d x^{n}$, and the meaning if $n=1 / 2$, and a reply from Leibniz "It will lead to a paradox a paradox from which one day useful consequences will be drawn, because there are no useless paradoxes". ${ }^{10}$ After time, Grünwald worked on the fractional operations in 1867. Riemann developed the theory of fractional integration and published his paper regarding this issue in 1892. Letnikov came after them and wrote several papers from 1868 to 1872 . For a time, Leibniz proved his idea about fractional calculus and fractional derivatives and integrals, which has seen explosive growth in many fields of science and engineering since then. The main reason for using integer-order models was the absence of solution methods for fractional differential equations.

At present, fractional calculus is used in many applications with the methods for the fractional derivative and integral approximation. For instance, mathematically, the historical background of anomalous diffusion in space and time domain using a variety of fractional-order derivatives was provided by Metzler and Klafter. ${ }^{11}$ The form and properties of fractional-order derivatives as mathematical description can be found in the monographs by Podlubny, ${ }^{12} \operatorname{Herrman}^{13}$ and Meerschaert and Sikorskii, ${ }^{14}$ while the theoretical models and experimental applications are available 
and published. ${ }^{15}$ Some applications are in fields that include bioengineering, ${ }^{16-19}$ filters ${ }^{20}$ control theory, ${ }^{21}$ signal processing, ${ }^{22}$ oscillators, ${ }^{23-29}$ transmission lines $(\mathrm{TLs})^{30-33}$ and potentially many others.

In comparison to classical integer-order models, fractional derivatives provide better results for the description of memory, materials and processes. Recently, there have been many engineering applications where the electronic circuits is one of the successful parts that show significantly good results for fabricating fractional-order capacitors by using silicon. Furthermore, the behavior of the three well-known elements: the resistor, the capacitor and the inductor are designed today in the time domain based on integer-order differential equations. Integer-order algebraic equations in the complex frequency $s$-domain are used to describe the linear dynamical systems via Laplace transform. Hence, the circuit order is directly proportional to the number of energy storage elements in the circuit.

Signal propagation on a RC TL can be described by a partial differential equation (PDE) that is equivalent in form to the equations describing the classical Gaussian diffusion. Fractional order generalizations of this model reflect the onset of anomalous, nonGaussian diffusion. Anomalous diffusion has been characterized in both space and time using a rich variety of fractional order derivatives when the line or skin effect is described by fractional differential equations. ${ }^{34}$ In recent decades, scientists and engineers recognize that the fractional differential equations provide a better approach to describe the complex phenomena in nature such as the skin effect, dielectric relaxation and chaos. In Ref. 35, Liu et al. considered the inherent fractional order characteristics of the skin effect and achieved a fractional order cable model using the Levy's fitting method $^{36}$ and the numerical solution for the fractional differential equations.

The idea of fractional derivatives and integrals is quite an interesting topic, very hard to explain, due to the fact that, unlike commonly used differential operators, it is not related to some important geometrical meaning. But, many physical phenomena have intrinsic fractional-order description and so fractional-order calculus is necessary to explain them. Therefore, in this paper, we tried to show the effect of fractional-orders on lossy TLs and multiconductor TLs (MTLs) at high frequencies. Comparing with the previous numerical solutions, the NILT method is used to solve system equations.

The rest of this paper is organized as follows: Sec. 2 briefly explains the fractionalorder lossy TL. Section 3 gives a deduction of the numerical solution for the fractional differential equations. Section 4 describes the wide-band modeling method of the MTLs considering the frequency-dependent parameters, Sec. 5 provides examples to verify the proposed algorithm, and finally the conclusion and discussion are presented in Sec. 6.

\section{Fractional-Order TL Model}

TL modeling in the time-domain is an ongoing challenge, especially with the continuous increase of operational frequencies and the decrease in feature size. 
Therefore, interconnects, integrated circuits and/or printed circuit boards are processed as systems with distributed parameters where the TL theory is considered. ${ }^{37-40}$ A typical lossy TL can be represented mathematically by a set of PDEs, namely, a pair of telegraphic equations. Introducing the Laplace transform, as it is well known, the TL system equations can be considerably simplified resulting in ordinary differential equations (ODEs). In the light of the above, considering zero initial voltage and current distributions, $v(0, x)=0$ and $i(0, x)=0$, respectively, and then performing the Laplace transform with respect to time variable $t$, we get $^{41}$

$$
\begin{gathered}
-\frac{d V(s, x)}{d x}=Z(s) I(s, x), \\
-\frac{d I(s, x)}{d x}=Y(s) V(s, x),
\end{gathered}
$$

where the per-unit-length (p.u.l.) series impedance and shunt admittance are given, respectively, as follows:

$$
\begin{aligned}
& Z(s)=R+s L, \\
& Y(s)=G+s C .
\end{aligned}
$$

with $R, L, C$ and $G$, as p.u.l. primary parameters, representing series resistance, series inductance, shunt capacitance and shunt conductance, accordingly. In Fig. 1 the Laplace-domain model of the TL with length $d$ is shown. This model corresponds to the solution of the system of Eqs. (1) and (2), while incorporating boundary conditions. The TL shown in Fig. 1 is terminated on both sides with linear networks; these linear networks can be represented by their Thévenin or Norton equivalents, here the former was considered. Considering this process, the results of the Laplacedomain solution are as follows:

$$
V(s, x)=V_{i}(s) \frac{Z_{c}(s)}{Z_{i}(s)+Z_{c}(s)} \cdot \frac{e^{-\gamma(s) x}+\rho_{2}(s) e^{-\gamma(s)[2 d-x]}}{1-\rho_{1}(s) \rho_{2}(s) e^{-2 \gamma(s) d}},
$$

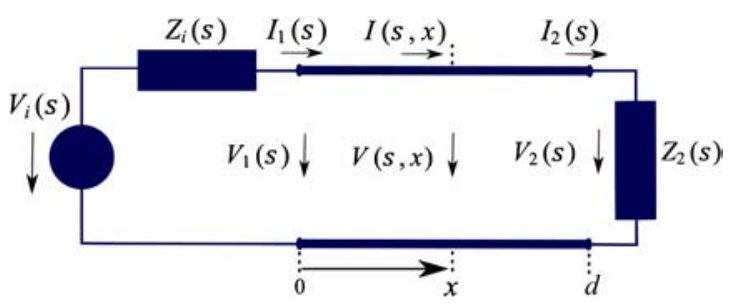

Fig. 1. Laplace-domain model of the TL with linear terminations. ${ }^{40}$ 


$$
I(s, x)=V_{i}(s) \frac{1}{Z_{i}(s)+Z_{c}(s)} \cdot \frac{e^{-\gamma(s) x}-\rho_{2}(s) e^{-\gamma(s)[2 d-x]}}{1-\rho_{1}(s) \rho_{2}(s) e^{-2 \gamma(s) d}}
$$

where $Z_{c}(s)$ and $\gamma(s)$ as the characteristic impedance and propagation constant, respectively, can be written as:

$$
Z_{c}(s)=\sqrt{\frac{Z(s)}{Y(s)}}, \quad \gamma(s)=\sqrt{Z(s) \cdot Y(s)} .
$$

The reflection coefficients $\rho_{1}(s)$ and $\rho_{2}(s)$ are described by the following equations:

$$
\rho_{1}(s)=\frac{Z_{i}(s)-Z_{c}(s)}{Z_{i}(s)+Z_{c}(s)}, \quad \rho_{2}(s)=\frac{Z_{2}(s)-Z_{c}(s)}{Z_{2}(s)+Z_{c}(s)} .
$$

In the following section the fractional-order elements (capacitance and inductance) are imposed to the TL model.

\subsection{Fractional-order elements}

Considering a TL with length $d$ and distributed elements as in Fig. 1, the elementary section of the TL can be shown as in Fig. 2. The primary parameters $L_{\alpha}, C_{\beta}$ have the fractional orders $\alpha$ and $\beta$, respectively; noting that the subscripts $\alpha, \beta$ represent only the fractional order immittances, while the numerical values of the parameters are not changed.

The main benefits of the fractional-order TLs are: having a high degree of freedom for TL modeling, enabling to realize the losses especially resulting from high frequencies, ${ }^{32}$ and furthermore, it builds on the fact that the solution of the TL traveling waveforms continuously depends on the fractional derivative.

Incorporating the fractional-order primary parameters into the TL systems equations results in

$$
Z_{c f}(s)=\sqrt{\frac{Z_{f}(s)}{Y_{f}(s)}}=\sqrt{\frac{R+s^{\alpha} L_{\alpha}}{G+s^{\beta} C_{\beta}}}
$$

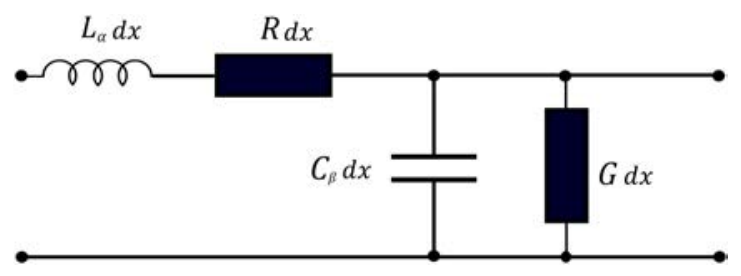

Fig. 2. Fractional-order primary parameters of the TL. ${ }^{38}$ 
where $Z_{c f}(s)$ is the fractional-order characteristic impedance, which is replaced instead of the integer-order characteristic impedance $Z_{c}(s)$ in (5).

Moreover, the propagation constant $\gamma(s)$ can be tuned accordingly with fractional parameters as

$$
\gamma_{f}(s)=\sqrt{Z_{f}(s) \cdot Y_{f}(s)}=\sqrt{\left(R+s^{\alpha} L_{\alpha}\right)\left(G+s^{\beta} C_{\beta}\right)} .
$$

Detailed analysis and discussions of the imposed fractional-order parameters will be shown in Sec. 4.

\subsection{Frequency dependence of the $T L$}

Frequency-dependent properties of the TL have a direct impact on the propagating signal waveforms, especially with the continuous rapid increase in the operational frequencies and the rise in the transmission speeds in power systems. Generally, the main frequency-dependent parameter to be considered is the skin effect as its impact on the TL is much higher than other frequency-dependent parameters, such as the polarization effect on the surrounding medium. ${ }^{42,43}$ As it is known, the skin effect increases the resultant losses of the TL. To feasibly incorporate the skin effect into the TL system of equations, Laplace transform is used rather than solving in the time-domain. The fractional-order series impedance is supplemented by the term $K \sqrt{s}$, to result in

$$
Z_{f}(s)=R+s^{\alpha} L_{\alpha}+K \sqrt{s}
$$

where the latter term $K \sqrt{s}$ represents high-frequency internal resistance including high frequency inductive reactance. Consequently, Eq. (11) is substituted in (9) and (10).

In the following section, a proposed NILT is introduced. This NILT method, recently developed by the authors, provides the possibility to obtain the time domain simulation result of the described TL system of equations.

\section{Analysis and Simulation Results}

Inspired by the authors work presented by Ismail et al. ${ }^{32}$ here we investigate the boundary regions for the choices of the fractional-orders $\alpha$ and $\beta$. Firstly, the analysis is done by observing the relation between the fractional-orders and the real part of the propagation constant $\gamma_{f}(s=j \omega)$ which represents the attenuation, and hence the sign should remain negative in the interval. Secondly, the relation between the fractions and the real part of the characteristic impedance $Z_{f}(s=j \omega)$, which represents the resistive element and hence its amplitude, is considered for positive regions. Hence, the theoretical general region is bounded as $0<\alpha, \beta<2$.

Consider a uniform lossy TL of length $d=1.2 \mathrm{~m}$ connected to an arbitrary generator $V_{i}(s)$ of impedance $Z_{i}=10 \Omega$ and to a load impedance $Z_{2}=2.5 \mathrm{k} \Omega$. The 
TL has the following parameters: $R_{0}=0.35 \Omega / \mathrm{m}, L_{\alpha}=265 \mathrm{nH} / \mathrm{m}, G=1 \mathrm{mS} / \mathrm{m}$, $C_{\beta}=95 \mathrm{pF} / \mathrm{m}$ and the skin effect parameter $K=2.9 \cdot 10^{-4} \Omega \sqrt{\mathrm{s}} / \mathrm{m}$ for the frequency, dependent line or $K=0$ is used for the frequency independent line. ${ }^{41}$ The fractional-order characteristic impedance with incorporating the skin effect is given as

$$
Z_{c f}(s)=\sqrt{\frac{R+s^{\alpha} L_{\alpha}+K \sqrt{s}}{G+s^{\beta} C_{\beta}}} .
$$

and consequently, the fractional-order propagation constant with the skin effect is given as

$$
\gamma_{f}(s)=\sqrt{\left(R+s^{\alpha} L_{\alpha}+K \sqrt{s}\right) \cdot\left(G+s^{\beta} C_{\beta}\right)} .
$$

The simulation results, illustrating the effect of variable fractional-orders $\alpha$ and $\beta$ on the real value of both $Z_{f}$ and $\gamma_{f}$, are shown in Figs. 3-8, accordingly. Namely, Figs. 3-6 illustrate the real value of $Z_{f}$ exposing the general regions which bound the

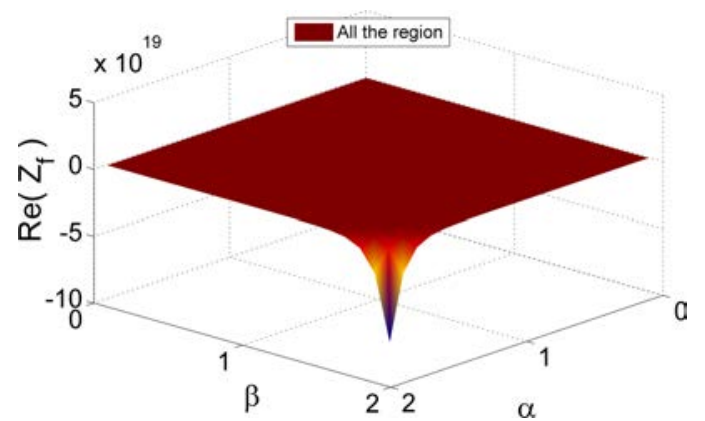

Fig. 3. Real $Z_{f}$ versus fractional-orders $\alpha$ and $\beta$ (all region).

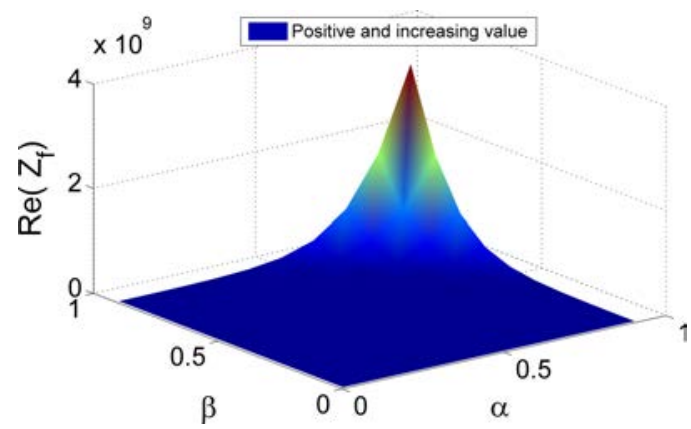

Fig. 4. Real $Z_{f}$ versus fractional-orders $\alpha$ and $\beta$ (region of interest). 


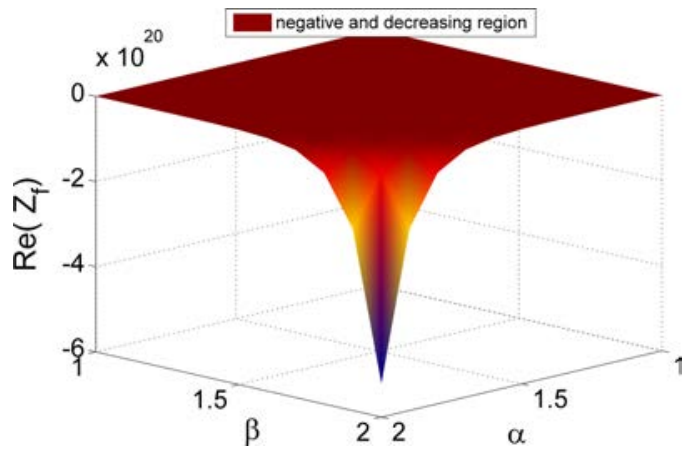

Fig. 5. Real $Z_{f}$ versus fractional-orders $\alpha$ and $\beta$ (region of interest).

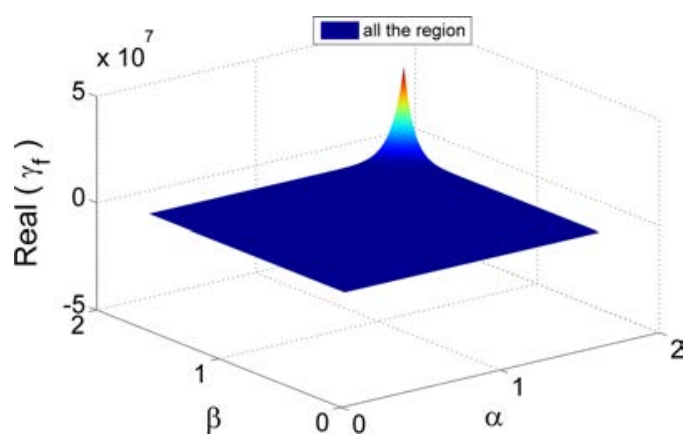

Fig. 6. Real $\gamma_{f}$ versus fractional-orders $\alpha$ and $\beta$ (all region).

selection choices of $\alpha, \beta$. The results were obtained by utilizing the Matlab programming language on high frequencies, by replacing the Laplacian operator $s$ with $\mathrm{j} \omega$ at $f=1 / T=0.5 \times 10^{9}$ and $\omega=2 \pi f=3.14 \times 10^{9} \mathrm{rad} \cdot \mathrm{s}^{-1}$ that is used in the simulation results below. Inspecting Fig. 3, it is interesting to notice the relation of

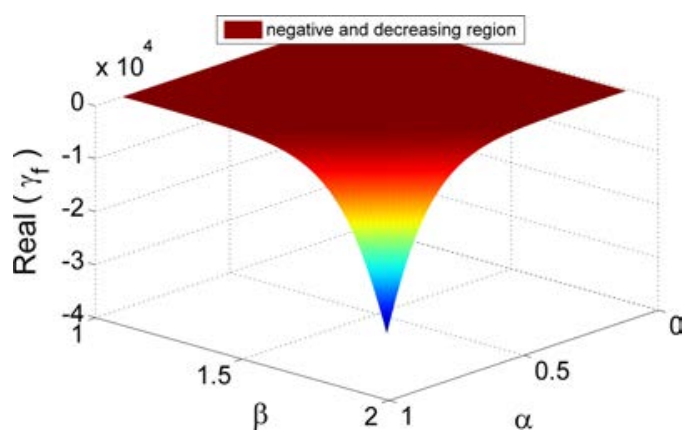

Fig. 7. Real $\gamma_{f}$ versus fractional-orders $\alpha$ and $\beta$ (region of interest). 


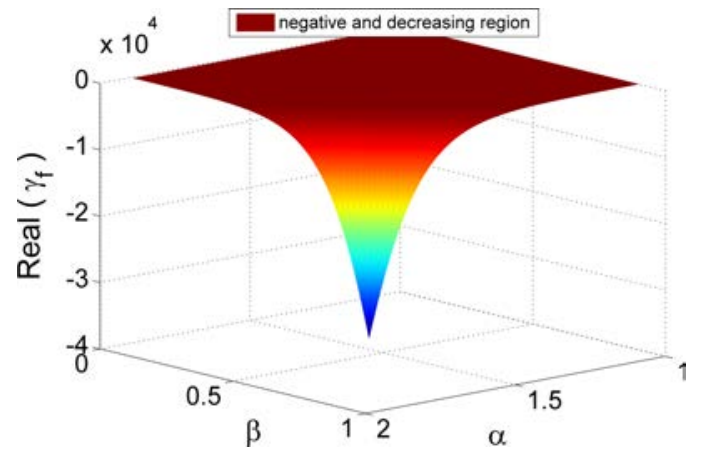

Fig. 8. Real $\gamma_{f}$ versus fractional-orders $\alpha$ and $\beta$ (region of interest).

the real part of $Z_{f}$ with the fractional-orders $\alpha$ and $\beta$, which gives us an insight on how the resistive element changes along the interval region with changing the fractional orders. Practically, with a linear arbitrary load, the response of a TL to an applied voltage signal is resistive rather than reactive, despite being theoretically composed of resistive, inductive and capacitive elements. In Figs. 3 and 6, the classical integer TL model can be noticed, i.e., at the point where $\alpha=\beta=1$. The following can be conceived from the simulation results of $Z_{f}$ and $\gamma_{f}$, namely, the real value of the $Z_{f}$, the resistive element, is positive and increasing when the fractional values are in the range $0<\alpha, \beta<1$. Hence, we bound our choices in this region; above this range the real value of the $Z_{f}$ is negative and decreasing as shown in Fig. 5.

Henceforth, after the fractional-order $L C$ elements are substituted to (3) and (4), and hence included in the TL system equations, the voltage and current waveforms can be then efficiently obtained, in the time-domain, by undergoing the proposed hyperbolic NILT method.

The hyperbolic NILT method, proposed in the authors' earlier works, ${ }^{6-8}$ is an effective and potential technique to numerically obtain the time-domain simulation of the TL from the frequency-domain system equations.

In concept, the method is devised based on approximating the exponential kernel $\exp (s t)$ in the definition Bromwich integral (14) by specific hyperbolic relations

$$
f(t)=\frac{1}{2 \pi j} \int_{c-j \infty}^{c+j \infty} F(s) e^{s t} d s,
$$

while the following is considered: $F(s)$ is regular for $\operatorname{Re}(s)>0$, when $|s| \rightarrow \infty$ then $F(s) \rightarrow 0$, and $F^{*}(s)=F\left(s^{*}\right)$, where the symbol * denotes a complex conjugate. To simplify the evaluation process of the integral, the exponential kernel is replaced 
by the following relations:

$$
E_{s}(s t, a)=\frac{e^{a}}{2 \sinh (a-s t)}, \quad E_{c}(s t, a)=\frac{e^{a}}{2 \cosh (a-s t)} .
$$

Furthermore, the reciprocal hyperbolic functions are replaced by their corresponding infinite series expansions, denoted by the variable $y$,

$$
\frac{1}{\sinh y}=\frac{1}{y}+2 y \sum_{n=1}^{\infty} \frac{(-1)^{n}}{n^{2} \pi^{2}+y^{2}}, \quad \frac{1}{\cosh y}=2 \pi \sum_{n=0}^{\infty} \frac{(-1)^{n}(n+0.5)}{(n+0.5)^{2} \pi^{2}+y^{2}},
$$

and then, with the subsequent application of the residual theorem, we obtain two approximate formulae

$$
\begin{gathered}
f_{s}(t, a)=\frac{e^{a}}{t}\left(\frac{1}{2} F\left(\frac{a}{t}\right)+\sum_{n=1}^{\infty}(-1)^{n} \operatorname{Re}\left\{F\left(\frac{a}{t}+j n \frac{\pi}{t}\right)\right\}\right), \\
f_{c}(t, a)=\frac{e^{a}}{t} \sum_{n=1}^{\infty}(-1)^{n} \operatorname{Im}\left\{F\left(\frac{a}{t}+j\left(n-\frac{1}{2}\right) \frac{\pi}{t}\right)\right\} .
\end{gathered}
$$

The theoretical absolute errors of (17) and (18) can be obtained by expressing the hyperbolic functions in (15) with their definition exponential functions and rearranging them in the form of the sum of infinite geometric series, see Ref. 6 for more details, we get, respectively,

$$
\varepsilon_{s}(t, a)=\sum_{n=1}^{\infty} e^{-2 n a} f((2 n+1) t), \quad \varepsilon_{c}(t, a)=\sum_{n=1}^{\infty}(-1)^{n} e^{-2 n a} f((2 n+1) t) .
$$

To further increase the accuracy an enhancement to the method was obtained by considering the arithmetic mean of (17) and (18), which gives a higher accuracy to the result or in other words a lower absolute error, the resulting formula and its absolute error are given as follows:

$$
\begin{aligned}
f_{\text {en }}(t, a)= & \frac{e^{a}}{2 t}\left(\frac{1}{2} F\left(\frac{a}{t}\right)+\sum_{n=1}^{\infty}(-1)^{n}\left(\operatorname{Re}\left\{F\left(\frac{a}{t}+j n \frac{\pi}{t}\right)\right\}\right.\right. \\
& \left.\left.+\operatorname{Im}\left\{F\left(\frac{a}{t}+j\left(n-\frac{1}{2}\right) \frac{\pi}{t}\right)\right\}\right)\right),
\end{aligned}
$$

with the absolute error as

$$
\varepsilon_{e n}(t, a)=\sum_{n=1}^{\infty} e^{-4 n a} f((4 n+1) t)
$$


It is evident that absolute error of the enhanced algorithm (21) is lower as the exponential is to the power of -4 when compared to power of -2 for the single approximations (19). In contrast to initial works, ${ }^{6,7}$ where Euler transformation was applied, here, the method has been further improved by incorporating the qd algorithm of Rutishauser to accelerate the convergence of the infinite series in (20) and providing a higher stability to the proposed NILT method, see Ref. 8 for more details. This hyperbolic qd-NILT method has been programed in the Matlab language and has been tested and analyzed, where it proves to perform with a high accuracy and computational effectiveness. ${ }^{44}$

In the following tests the TL in Fig. 1 is excited by the following voltage waveform:

$$
v_{i}(t)= \begin{cases}\sin ^{2}\left(\frac{\pi \cdot t}{2 \cdot 10^{-9}}\right), & \text { for } 0<t<2 \cdot 10^{-9} \\ 0, & \text { elsewhere }\end{cases}
$$

with the following Laplace domain transform:

$$
V_{i}(s)=\frac{2 \pi^{2}\left(1-\exp \left(-2 \cdot 10^{-9} s\right)\right)}{s\left(\left(2 \cdot 10^{-9} s\right)^{2}+4 \pi^{2}\right)} .
$$

The voltage/current waveforms are simulated for three cases: the integer TL model, the integer TL model with frequency-dependent parameters and the fractionalorder TL model with frequency-dependent parameters. Results are illustrated in Figs. 9 and 10.

It is clearly seen from the simulation results of Figs. 9 and 10 that the fractionalorder model including skin effect (in green) illustrates the losses on the TL more precisely than by using the classical integer-order model (in red). Nevertheless, the flexibility of the choices of the fractional-orders, in the boundary range, could be further optimized when comparing theoretical results to the practically measured results of a TL. Noticeably, the voltage/current waveform propagations are strongly

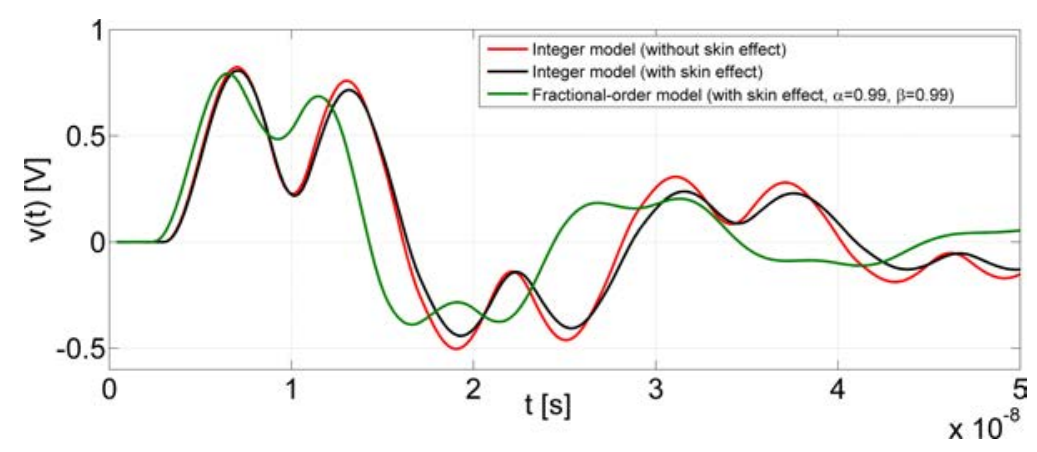

Fig. 9. Voltage waveform simulations in the middle of the TL $(x=0.6 \mathrm{~m})$. 


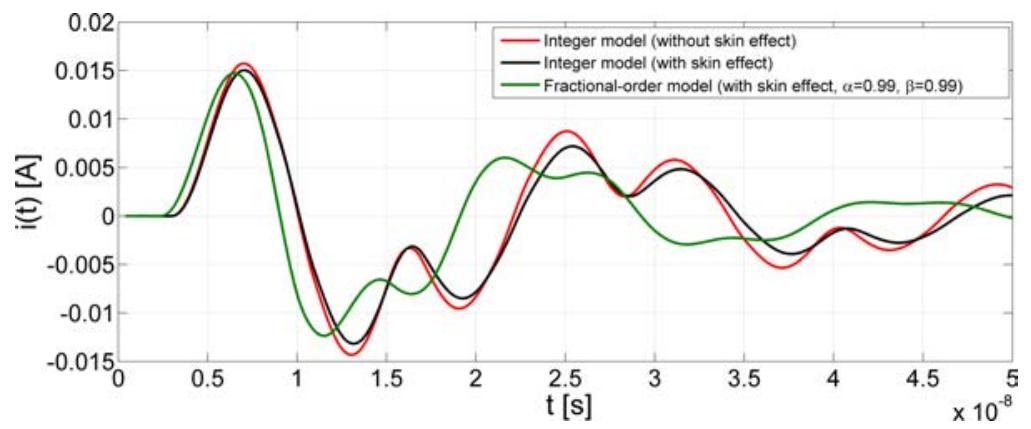

Fig. 10. Current waveform simulations in the middle of the TL $(x=0.6 \mathrm{~m})$.

affected by the skin effect (Figs. 9 and 10, in black). In fact, as it is well known, the skin effect influences the TL by decreasing the cross-sectional area; therefore, it affects the resistance of the conductor by increasing it. As a result, the skin effect causes a TL to heat up faster and to higher temperatures at higher frequencies, though with the same levels of current. ${ }^{45}$ Consequently, it is of importance to include frequency dependence parameters, especially with the increase in operational frequencies. Regarding the fractional-order parameters $\alpha$ and $\beta$, it is easily noticed from Figs. 9 and 10, in green, even with a small change in the fractional-order (i.e., 0.99) that the results are significant. It is important to note that the fractional-order modeling provides us with a higher degree of freedom for effectively modeling TL.

In addition, another approach for the simulation of voltage and current distributions on the TL can be performed by further transforming the telegraphic equations not only with respect to time $t$, as in (1) and (2), but also with respect to the geometric coordinate $x$, resulting with purely algebraic equations. Namely, after performing the Laplace transform for both variables, incorporating boundary conditions and similarly considering zero initial voltage and current distributions, we get as result ${ }^{41}$

$$
\begin{gathered}
V(s, q)=\frac{q V_{1}(s)-\gamma(s) Z_{c}(s) I_{1}(s)}{q^{2}-\gamma^{2}(s)} \\
I(s, q)=\frac{q I_{1}(s)-\frac{\gamma(s)}{Z_{c}(s)} V_{1}(s)}{q^{2}-\gamma^{2}(s)} .
\end{gathered}
$$

Fractional-order parameters can be incorporated by similar way as previously, by replacing the characteristic impedance $Z_{c}(s)$ and the propagation constant $\gamma(s)$ with (12) and (13). Then, after determining boundary conditions $V_{1}(s)$ and $I_{1}(s)$, the time-spatial domain voltage and current waveforms of (24) and (25) can be easily obtained by undergoing the 2D FFT NILT method, ${ }^{41}$ as shown in Figs. $11-13$. 
In Figs. 11-13, the voltage distributions along the TL wires are illustrated. The results show three different cases; namely, the integer model, i.e., $\alpha=\beta=1$, for fractional-orders of $\alpha=\beta=0.98$ and for that of $\alpha=\beta=0.96$, respectively. These fractional values are all selected to be in the range boundary described earlier in the paper. It is evident that when $\alpha, \beta=0.96$ the diffusion is faster

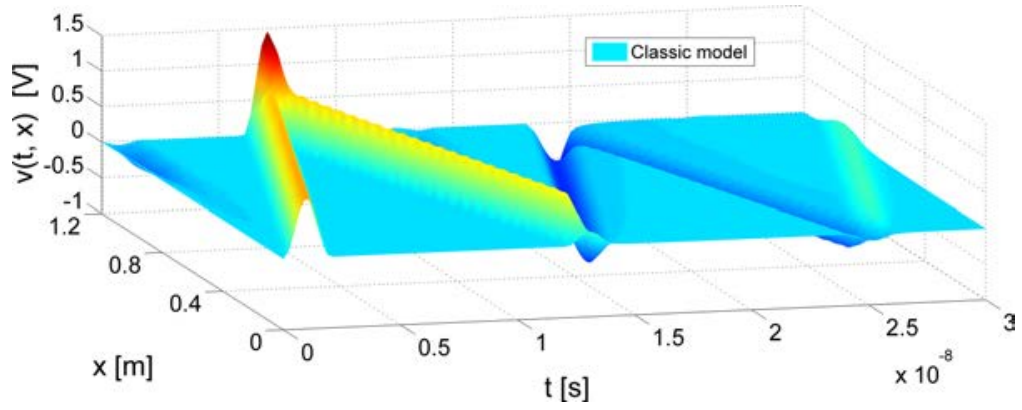

Fig. 11. Voltage waveform along the lossy TL with integer-order elements.

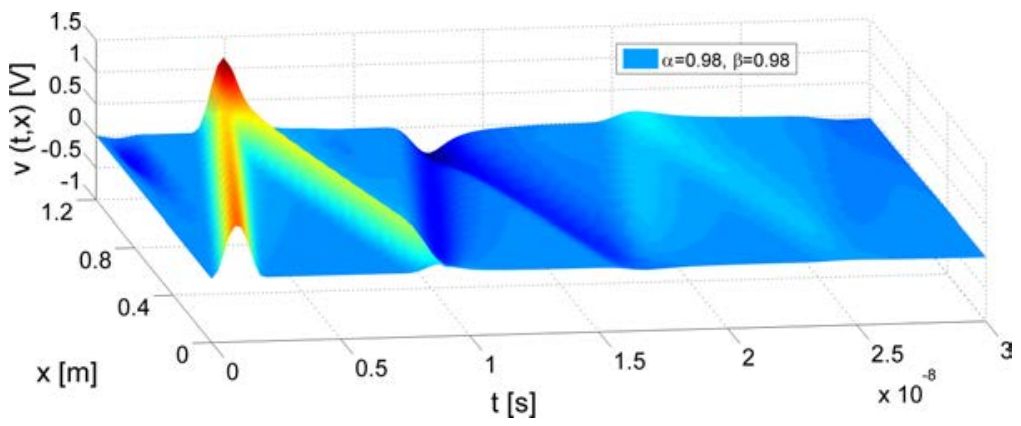

Fig. 12. Voltage waveform along the lossy TL with fractional-order elements $\alpha=\beta=0.98$.

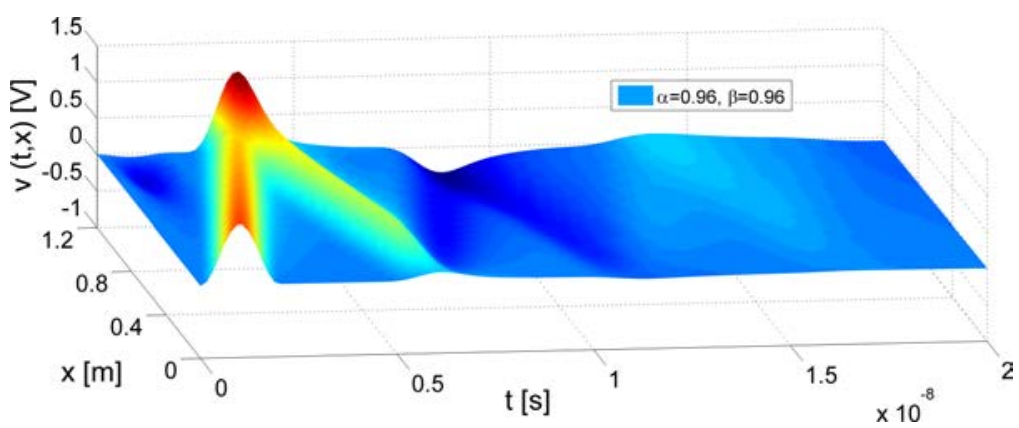

Fig. 13. Voltage waveform along the lossy TL with fractional-order elements $\alpha=\beta=0.96$. 
than when $\alpha, \beta=0.98$, such behaviors of fractional parameters were also observed in Ref. 33.

These results are generally consistent with the behavior of fractional-order systems. Essentially, as the incident and reflected waves of the TL travel on opposite directions, this, with time, leads to a reduction in the spread of voltage waveform; specially, this builds on the fact that the solution continuously depends on the fractional derivative. As a result, the following can be conceived, the fractional-order TL model captures the abnormal diffusion phenomena of the voltage/current waves traveling along the TL much more precisely than the classical integer model, as noted in Refs. 33 and 46. The incorporation of fractional-order elements in TL modeling provide us with various benefits; for example, it captures the phenomena and properties that classical integer-order models neglect. Generally, the modeling of the distributed parameter systems dynamics by using fractional calculus is a useful tool due to its infinite dimensionality. ${ }^{47}$ A practical measurement of a lossy semi-infinite fractional order TL model considering fractional $R$ and $C$ parameters was presented in Ref. 48, and it demonstrates fractional-order behavior, where the current into the line is equal to the half-order derivative of the applied voltage.

The simulation results shown in Figs. 9 and 10 were computed using the hyperbolic 1D NILT method accelerated with the qd algorithm ${ }^{8}$; whereas, the $3 \mathrm{D}$ voltage distributions in Figs. $11-13$ were obtained using the 2D FFT NILT method, ${ }^{41}$ these methods were developed and/or enhanced by the authors. The computation time for obtaining the voltage waveform results in Fig. 9 were on average of $550 \mathrm{~ms}$ on a PC with $2.8 \mathrm{GHz} / 8 \mathrm{~GB}$.

\section{Fractional-Order MTL Model}

MTLs are often found in communication systems, power distribution systems and digital computers. Hence, the analysis of such systems seems to be of high practical importance. In Sec. 3, we examined the time-domain analysis of the TL system equations and incorporated with frequency dependences and fractional-order elements. In this section, we will focus on MTLs, which consist of more than two conductors. It will be shown that the process to expand the result obtained for the TL to the MTL is straightforward, mainly done by introducing the matrix notation. Generally, there are several methods that can be considered when simulating MTL, such as the derivation of the MTL equations from the integral form of Maxwells equations, derivation of the MTL equations from the p.u.l. equivalent circuit, utilizing the "implicit Wendroff and state variable" method and others. ${ }^{42,49-52}$

The main attention will be focusing on MTLs with linear network terminations. As it will be shown in further details, these terminal networks can be modeled by the generalized Thévenin or Norton equivalents. The intention is not only to obtain the voltage and current waveforms at the MTLs ends, but to further illustrate how the voltage/current waves are propagating along the MTL. The presented MTL 
model differs from the classic one by incorporating fractional-order elements. This is made feasible by the advantage of utilizing the NILT methods.

In general, let us consider an $(n+1)$-conductor of uniform TL with length $d$ and p.u.l. $n \times n$ matrices $\mathbf{R}, \mathbf{L}, \mathbf{G}$ and $\mathbf{C}$.

First, we start with a classical MTL description, afterwards fractional-order elements will be incorporated. For a uniform $(n+1)$-conductor TL, if zero initial conditions are considered, i.e., $\mathbf{v}(0, x)=\mathbf{0}$, and $\mathbf{i}(0, x)=0$, the Laplace-domain telegraph equations in a matrix form are

$$
\frac{d}{d x}\left[\begin{array}{c}
\mathbf{V}(s, x) \\
\mathbf{I}(s, x)
\end{array}\right]=\left[\begin{array}{cc}
\mathbf{0} & -\mathbf{Z}(s) \\
-\mathbf{Y}(s) & \mathbf{0}
\end{array}\right] \cdot\left[\begin{array}{l}
\mathbf{V}(s, x) \\
\mathbf{I}(s, x)
\end{array}\right]
$$

with the $n \times 1$ voltage and current vectors $\mathbf{V}(s, x)=\mathbf{L}\{\mathbf{v}(t, x)\}$ and $\mathbf{I}(s, x)=$ $\mathbf{L}\{\mathbf{i}(t, x)\}$, respectively, and $\mathbf{0}$ standing for the $n \times n$ order zero matrix, a series impedance matrix and a shunt admittance matrix given, respectively, as follows:

$$
\begin{gathered}
\mathbf{Z}(s)=\mathbf{R}+s \mathbf{L}, \\
\mathbf{Y}(s)=\mathbf{G}+s \mathbf{C} .
\end{gathered}
$$

When considering the boundary condition at $x=0, \quad \mathbf{V}_{1}(s)=\mathbf{V}(s, 0)$ and $\mathbf{I}_{1}(s)=\mathbf{I}(s, 0)$, see Fig. 14, a formal solution can be stated as

$$
\left[\begin{array}{l}
\mathbf{V}(s, x) \\
\mathbf{I}(s, x)
\end{array}\right]=\exp \left(\left[\begin{array}{cc}
\mathbf{0} & -\mathbf{Z}(s) \\
-\mathbf{Y}(s) & \mathbf{0}
\end{array}\right] \cdot x\right) \cdot\left[\begin{array}{c}
\mathbf{V}_{1}(s) \\
\mathbf{I}_{1}(s)
\end{array}\right]
$$

When considering the MTL as a linear multiport, the above matrix exponential function can be considered as a chain matrix, namely,

$$
\Phi(s, x)=\left[\begin{array}{ll}
\Phi_{11}(s, x) & \Phi_{12}(s, x) \\
\Phi_{21}(s, x) & \Phi_{22}(s, x)
\end{array}\right]=\exp \left(\left[\begin{array}{cc}
\mathbf{0} & -\mathbf{Z}(s) \\
-\mathbf{Y}(s) & \mathbf{0}
\end{array}\right] \cdot x\right)
$$

where $\Phi_{i j}(s, x), i, j=1,2$, are respective $n \times n$ square submatrices. Now, for simplicity, let us designate the chain matrix of the whole MTL of the length $d$ as

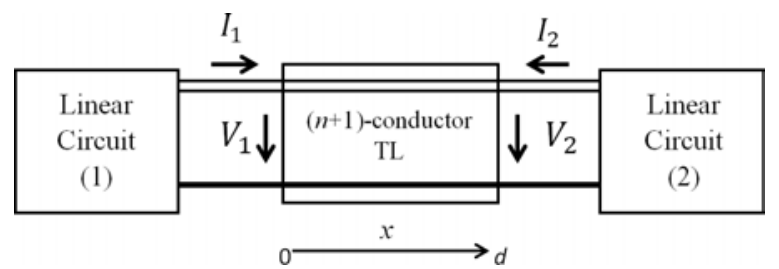

Fig. 14. General $(n+1)$-conductor TL system with linear terminations. ${ }^{51}$ 
$\Phi(s, d)=\Phi(s)$. Then, by using e.g., a generalized Thévenin equivalents of both terminating linear circuits the formulae for the boundary vectors $\mathbf{I}_{1}(s)$ and $\mathbf{V}_{1}(s)$ can be determined as

$$
\begin{gathered}
\mathbf{I}_{1}(s)=\left[\left(\Phi_{11}(s)-\mathbf{Z}_{i 2}(s) \Phi_{21}(s)\right) \mathbf{Z}_{i 1}(s)+\mathbf{Z}_{i 2}(s) \Phi_{22}(s)-\Phi_{12}(s)\right]^{-1} \\
\times\left[\left(\Phi_{11}(s)-\mathbf{Z}_{i 1}(s) \Phi_{21}(s)\right) \mathbf{V}_{i 1}(s)-\mathbf{V}_{i 2}(s)\right] \\
\mathbf{V}_{1}(s)=\mathbf{V}_{i 1}(s)-\mathbf{Z}_{i 1}(s) \mathbf{I}_{1}(s)
\end{gathered}
$$

where $\mathbf{V}_{i 1}(s), \mathbf{V}_{i 2}(s)$ and $\mathbf{Z}_{i 1}(s), \mathbf{Z}_{i 2}(s)$ are internal voltage vectors and internal impedance matrices of the respective Thévenin equivalents, respectively. Similarly, further combinations of Thévenin and/or Norton equivalents can be considered for both sides of the MTL.

Incorporating fractional-order elements resides in interchanging the p.u.l. series impedance $\mathbf{Z}(s)$ and shunt admittance $\mathbf{Y}(s)$ matrices with $\mathbf{Z}_{f}(s)$ and $\mathbf{Y}_{f}(s)$, respectively, as follows:

$$
\begin{gathered}
\mathbf{Z}_{f}(s)=\mathbf{R}+s^{\alpha} \mathbf{L}_{\alpha}, \\
\mathbf{Y}_{f}(s)=\mathbf{G}+s^{\beta} \mathbf{C}_{\beta} .
\end{gathered}
$$

Similarly as in a single TL, a skin effect can be incorporated by further modification of the series impedance (33) with the term $\mathbf{K} \sqrt{s}$, though with a matrix coefficient $\mathbf{K}$. A second approach to solve the MTL system equations can be performed by further applying another Laplace transform with respect to the geometric coordinate $x,{ }^{53}$ while considering fractional-order MTL elements, which leads to the following matrix equation in the $(s, q)$ domain:

$$
\left(\begin{array}{c}
\mathbf{V}(s, q) \\
\mathbf{I}(s, q)
\end{array}\right)=\left(\begin{array}{cc}
q \mathbf{E} & \mathbf{Z}_{f}(s) \\
\mathbf{Y}_{f}(s) & q \mathbf{E}
\end{array}\right)^{-1} \cdot\left(\begin{array}{c}
\mathbf{V}_{1}(s) \\
\mathbf{I}_{1}(s)
\end{array}\right)
$$

where $\mathbf{E}$ is the identity matrix, and the voltage and current vectors corresponding to the left side of the line are obtained assuming generalized Thévenin equivalents considered for both terminating linear circuits, as shown in (32) and (31), respectively. Consequently, the time domain results can be obtained by undergoing the FFT 2D NILT method in a fast and accurate process. ${ }^{54}$

\section{Fractional-Order MTL Simulation Results}

As an example, we will consider a $(3+1)$-conductor TL as shown if Fig. 15, with length $d=1.2 \mathrm{~m}$. The MTL has the following terminal resistors $R_{1 i}=R_{2 i}=1 \Omega$, 


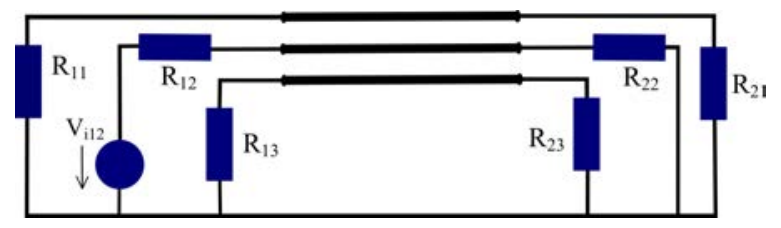

Fig. 15. The $(3+1)$-conductor TL system.

where $i=1,2,3$. The MTL's p.u.l. matrices are as follows ${ }^{55}$ :

$$
\begin{gathered}
\mathbf{R}=\left(\begin{array}{ccc}
41.7 & 0 & 0 \\
0 & 41.7 & 0 \\
0 & 0 & 41.7
\end{array}\right) \frac{\Omega}{\mathrm{m}}, \quad \mathbf{L}_{\alpha}=\left(\begin{array}{ccc}
2.4 & 0.69 & 0.64 \\
0.69 & 2.36 & 0.69 \\
0.64 & 0.69 & 2.4
\end{array}\right) \frac{\mu H}{\mathrm{~m}} \\
\mathbf{C}_{\beta}=\left(\begin{array}{ccc}
21 & -12 & -4 \\
-12 & 26 & -12 \\
-4 & -12 & 21
\end{array}\right) \frac{\mathrm{pF}}{\mathrm{m}}, \quad \mathbf{G}=\left(\begin{array}{ccc}
0.6 & 0 & 0 \\
0 & 0.6 & 0 \\
0 & 0 & 0.6
\end{array}\right) \frac{\mathrm{mS}}{\mathrm{m}}
\end{gathered}
$$

The voltage source $V_{i 12}$ driving the second wire in Fig. 15 is excited by the waveform (22). As the MTL is considered to have zero initial conditions, it is thus not required to compute the sophisticated convolution integrals. ${ }^{54}$ The results are directly obtained in the time domain by using the 1D NILT method based on FFT and the qd algorithm in matrix form. ${ }^{56}$

The simulation results of the voltage/current propagations are illustrated in Figs. 16-21 for three cases; namely, the integer case $\alpha=\beta=1$, for fractions-order case $\alpha=\beta=0.97$ and for the case where $\alpha=\beta=0.84$, respectively. Figures 16-27 show the voltage and current waveform distribution along the MTL wires for $\alpha$ and $\beta=0.84,0.97$ and 1 . Figs. 16, 19, 22 and 25 show the solutions of voltage/current waveforms for the classic MTL. It can be seen when comparing the MTL simulation

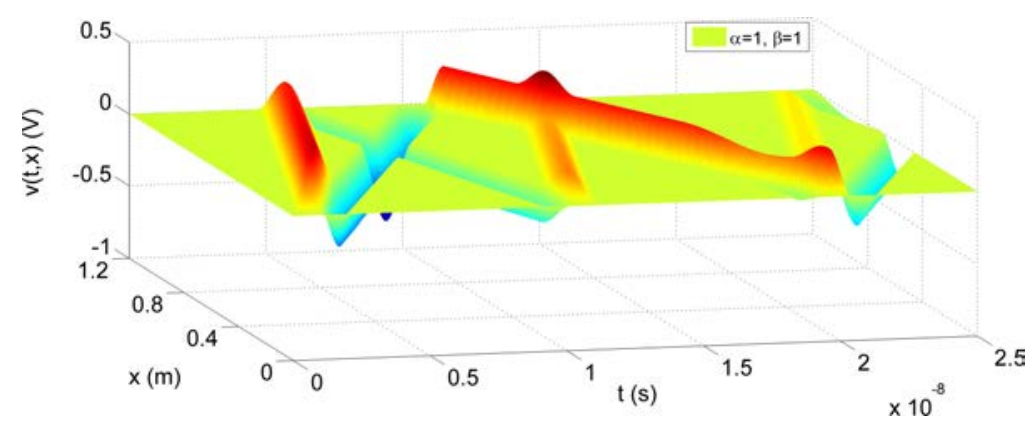

Fig. 16. Voltage distribution on the 1 st and the 3rd line with orders $\alpha=\beta=1$. 


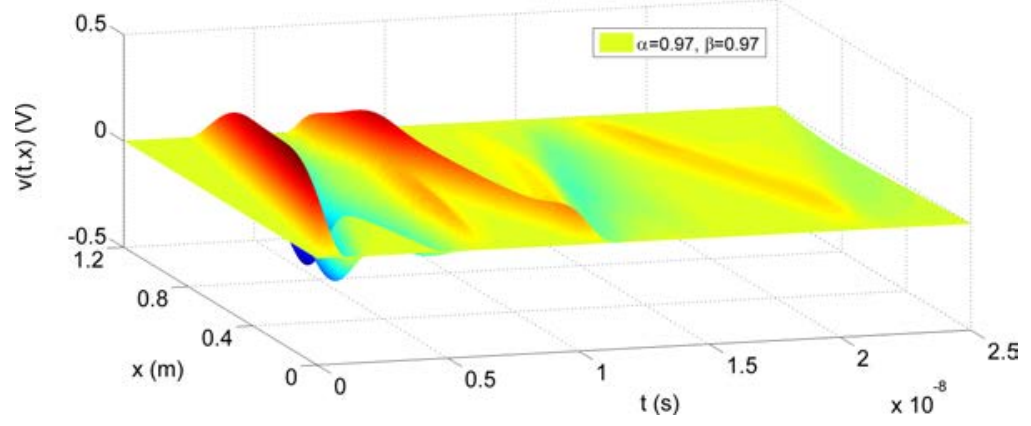

Fig. 17. Voltage distribution on the 1st and the 3rd line with orders $\alpha=\beta=0.97$.

results, shown in Figs. 16-27, that the increase of the fractional-orders from 0.84 to 0.97 influences the propagating waveform by exhibiting a faster wave spread at the beginning of the time interval; this agrees with the results of the TL simulations obtained in Sec. 3 and with the diffusion theory investigated in Refs. 33 and 45.

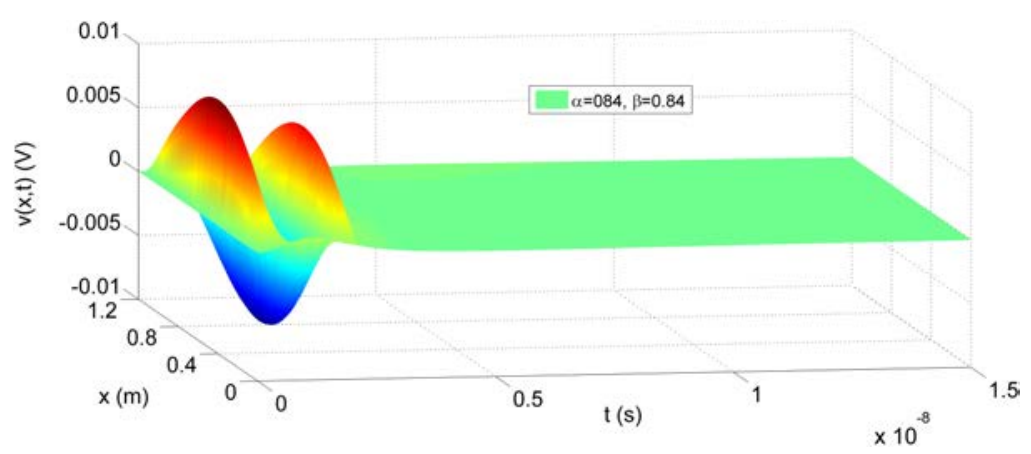

Fig. 18. Voltage distribution on the 1st and the 3rd line with orders $\alpha=\beta=0.84$.

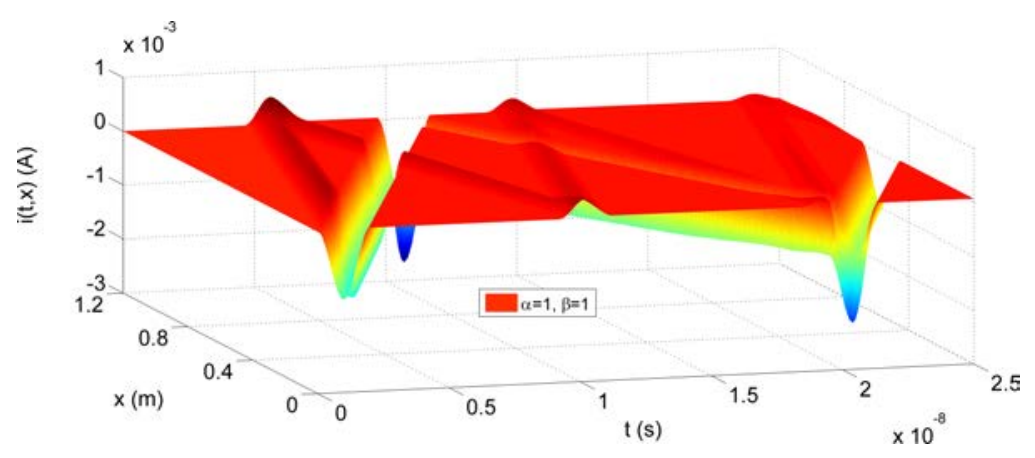

Fig. 19. Current distribution on the 1st and the 3rd line with orders $\alpha=\beta=1$. 


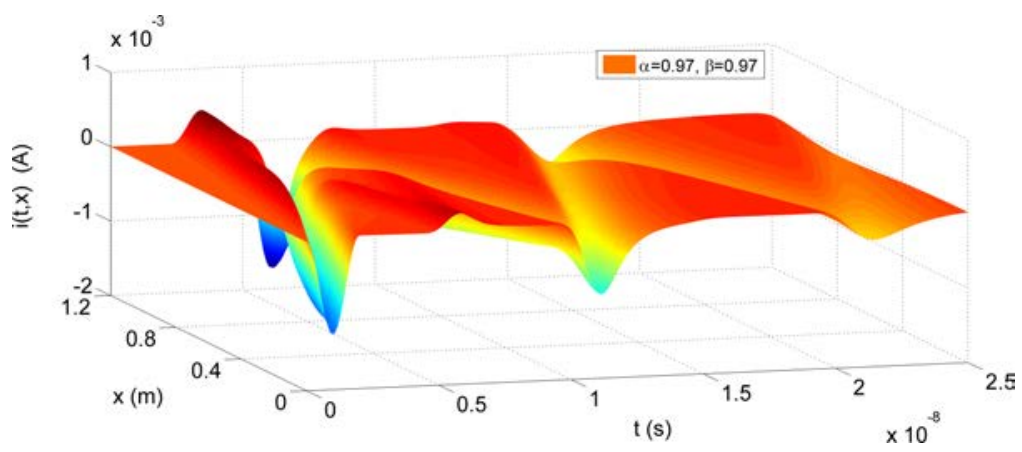

Fig. 20. Current distribution on the 1st and the 3rd line with orders $\alpha=\beta=0.97$.

Moreover, observing the results in the case when introducing fractional-orders, such as a comparison between Figs. 25 and 26, it shows that the waveform exhibits more attenuation along the wire and hence compensates for power losses and gives a higher degree for optimization.

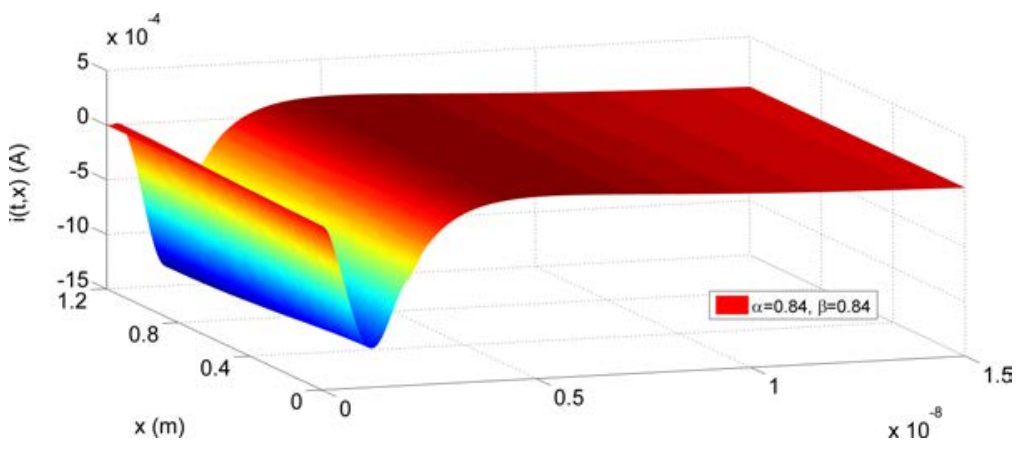

Fig. 21. Current distribution on the 1st and the 3rd line with orders $\alpha=\beta=0.84$.

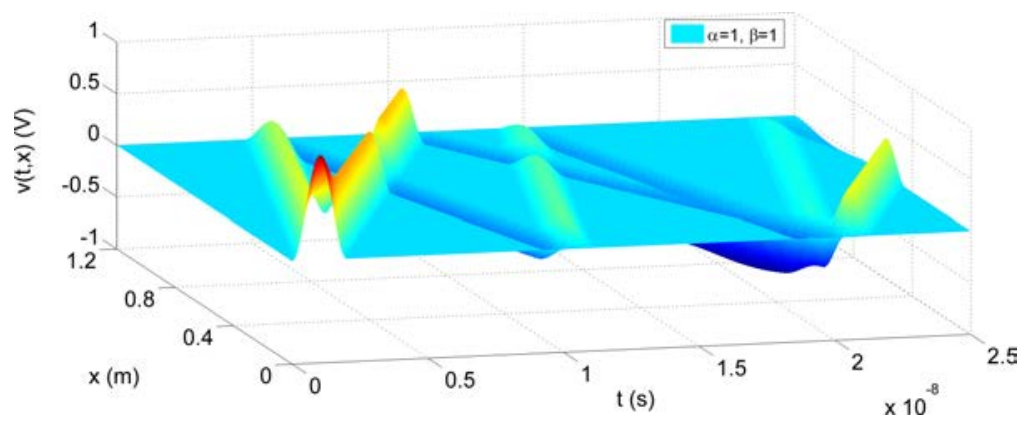

Fig. 22. Voltage distribution on the 2nd line with orders $\alpha=\beta=1$. 


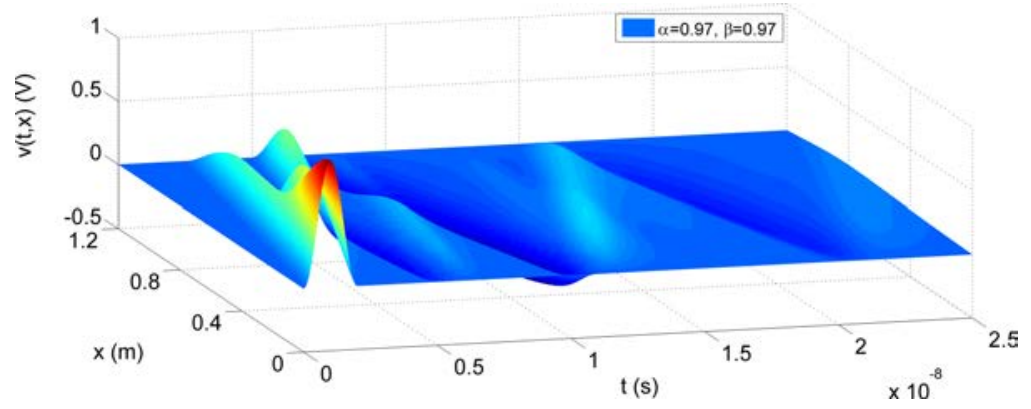

Fig. 23. Voltage distribution on the 2nd line with orders $\alpha=\beta=0.97$.

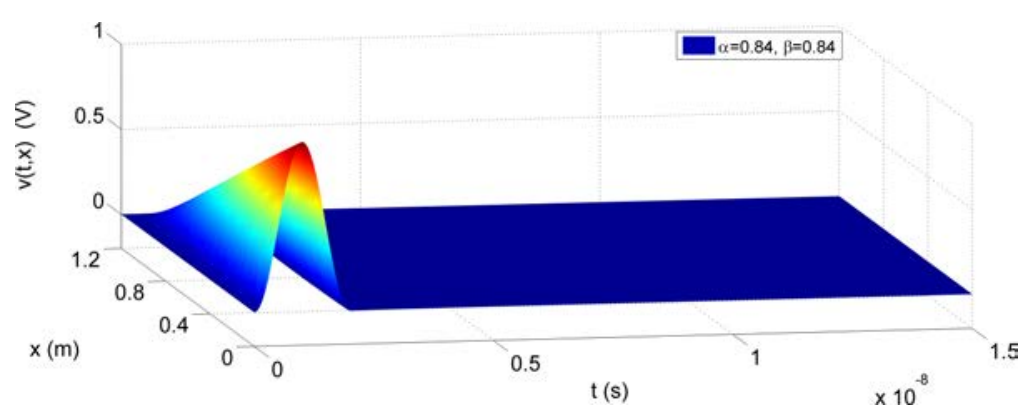

Fig. 24. Voltage distribution on the 2nd line with orders $\alpha=\beta=0.84$.

Looking into Figs. 23 and 24, it is noticed that the superposition effect that happens on TL wires when the forward wave and reflected wave interact is obvious in these two figures; this further shows that the physical phenomenon of MTL is more accurately modeled using fractional-order techniques.

A main advantage of utilizing the FFT 1D NILT accelerated by the qd algorithm, ${ }^{56}$ in its matrix form, can be noticed from the obtained MTL results. The

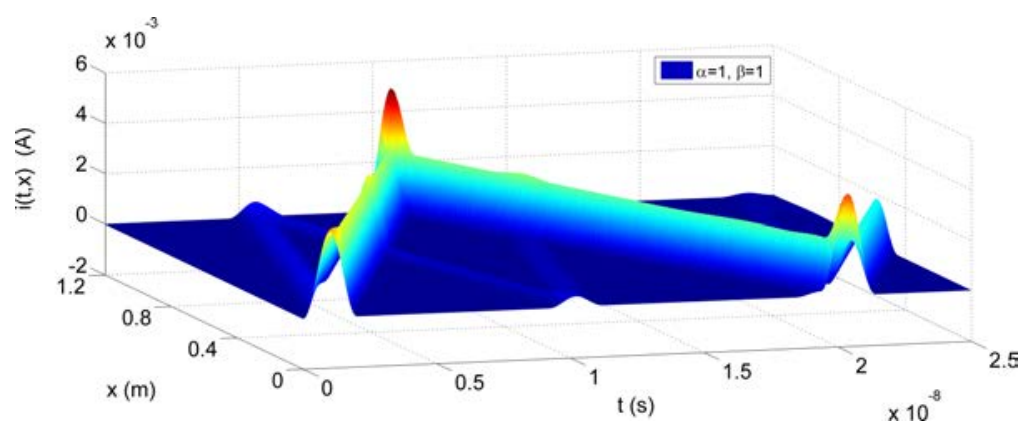

Fig. 25. Current distribution on the 2nd line with orders $\alpha=\beta=1$. 


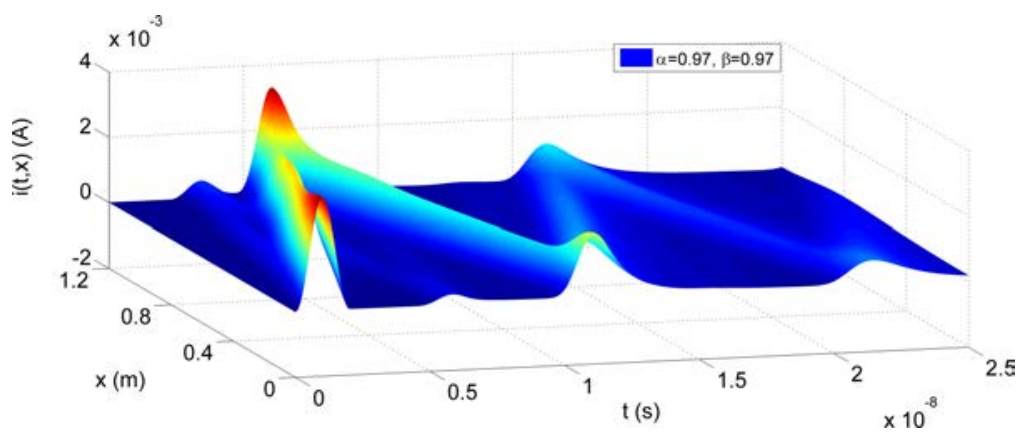

Fig. 26. Current distribution on the 2nd line with orders $\alpha=\beta=0.97$.

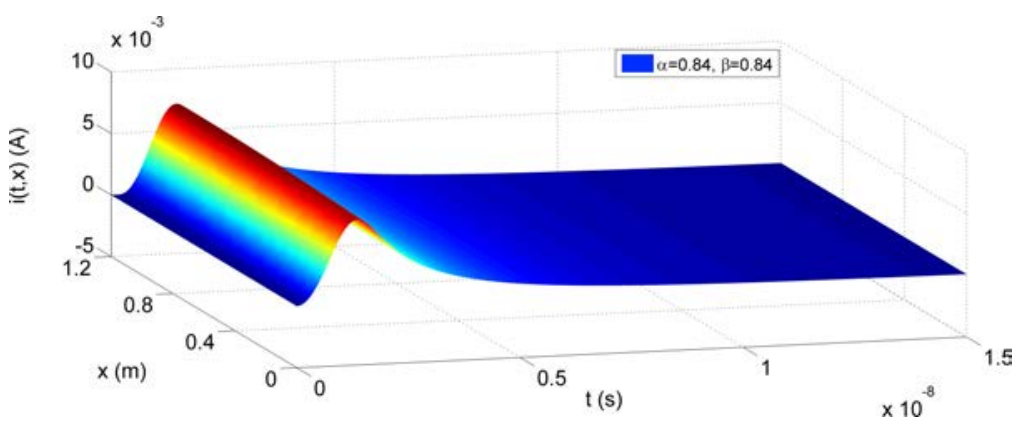

Fig. 27. Current distribution on the 2nd line with orders $\alpha=\beta=0.84$.

method allows us to solve the waves on all the MTL's wires in parallel. Depending on the defined resolution of the results and the number of terms considered for the FFT 1D NILT computations, the average CPU time for the simulations is approximately $4-6$ s running on a $\mathrm{PC}$ with $2.8 \mathrm{GHz} / 8 \mathrm{~GB}$.

In principle, the FFT 1D NILT method is devised based on computing the ILT, of Bromwich definition integral, numerically on a whole given interval $\left(0: t_{m}\right)$. The original approach of this method was proposed in Ref. 1, where the FFT algorithm is applied in order to provide the solution in an accurate and fast way. Moreover, further improvements have been done on this method, such as incorporating acceleration techniques and a development into a matrix form.

\section{Conclusion}

In this paper, a method for modeling and simulating for some distributed systems, based on the NILT method, has been described to evaluate the time-domain solution of voltage/current waveforms. The main advantage of using this approach is to obtain the whole $2 \mathrm{D}$ interval of the solution in a relatively fast and simple manner. 
Specifically, in this paper, the TL and MTL are realized by a pragmatic approach incorporating skin effect and fractional-order primary parameters. The choices of the fractional-orders used for the simulation were optimized by the analysis of their variations with the real parts of the characteristic impedance and the propagation constant separately. Generally, the results are noticed to be more realistic, also maintaining the authenticity of real conducting lines. Moreover, the incorporation of fractional-order modeling gives us a higher degree of freedom for optimization and compensates power losses along the lines. The $2 \mathrm{D}$ results for the TL are obtained by undergoing the hyperbolic NILT method; whereas the MTL time-domain solutions are obtained by utilizing the generalized matrix form of the NILT method based on FFT and qd algorithm, which shows to be an efficient method for inverting Laplace transforms in matrix forms.

\section{Acknowledgments}

This research was financially supported by the Czech Science Foundation under Grant 15-18288S. For research requirements, infrastructure of the SIX Center was used.

*A preliminary version of this paper has been presented at the 2017 40th International Conference on Telecommunications and Signal Processing (TSP). ${ }^{40}$

\section{References}

1. L. Brančík, The fast computing method of numerical inversion of Laplace transforms using FFT algorithm, Proc. 5th EDS'98 Int. Conf., Brno, Czech Republic (1998), pp. 97-100.

2. L. Brančík, An improvement of FFT-based numerical ILT procedure by application of $\epsilon$-algorithm, Proc. Sborník přednašek Moderni směry vyuky elektrotechniky a elektroniky STO-7, Brno, Czech Republic (1999), pp. 196-199.

3. L. Brančík and J. Valsa, A fast computing method of numerical inversion of twodimensional Laplace transforms using FFT algorithms, Proc. Advances Systems, Signals, Control and Computers SSCC'98, Durban, South Africa (1998), pp. 102-106.

4. L. Brančík, An improvement of FFT-based numerical inversion of two-dimensional Laplace transforms by means of $\epsilon$-algorithm, ISCAS-IEEE Int. Symp. Circuits and Systems, Geneva, Switzerland (2000), pp. 581-584.

5. L. Brančík, Numerical inversion of two-dimensional Laplace transforms based on partial inversions, Proc. 17th Int. Conf. Radioekektronika, Brno, Czech Republic (2007), pp. 194-197.

6. J. Valsa and L. Brančík, Approximate formulae for numerical inversion of Laplace transforms, Int. J. Numer. Model. Electron. Netw. Devices Fields 11(3) (1998) $153-166$.

7. L. Brančík and N. A.-Z. R-Smith, Two approaches to derive approximate formulae of NILT method with generalization, 38th Int. Conf. Information and Communication Technology MIPRO, Opatija, Croatia (2015), pp. 155-160. 
8. N. A.-Z. R-Smith and L. Brančík, Convergence acceleration techniques for proposed numerical inverse Laplace transform method, 24th Telecommunications Forum TELFOR (Belgrade, Serbia, 2016), pp. 1-4.

9. K. Oldham and J. Spanier, The Fractional Calculus: Theory and Applications of Differentiation and Integration to Arbitrary Order (Academic Press, New York, 1974).

10. M. Ortigueira, Fractional Calculus for Scientists and Engineers (Springer, 2011).

11. R. Metzler and J. Klafter, The random walk's guide to anomalous diffusion: A fractional dynamics approach, Physics Rep. 339 (2000) 1-77.

12. I. Podlubny, Fractional Differential Equations: An Introduction to Fractional Derivatives, Fractional Differential Equations, Some Methods of Their Solution and Some of Their Applications (Academic press, New York, 1999).

13. R. Hermann, Fractional Calculus an Introduction for Physicists (World Scientific, Singapore, 2011).

14. M. Meerschaert and A. Sikorskii, Stochastic Models for Fractional Calculus. (De Gruyter, Germany, 2011).

15. R. Klages, G. Radons and I. M. Sokolov, Anomalous Transport: Foundations and Applications (Wiley-VCH, Germany, 2008).

16. R. E. Gutierrez, J. M. Rosario and J. T. Machado, Fractional order calculus: Basic concepts and engineering applications, Math. Probl. Eng. 2010 (2010) Article ID $3758581-19$.

17. N. Sebaa, Z. E. A. Fellah, W. Lauriks and C. Depollier, Application of fractional calculus to ultrasonic wave propagation in human cancellous bone, Signal Proc. 86 (2006) $2668-2677$.

18. S. Abbisso, R. Caponetto, O. Diamante, L. Fortuna and D. Porto, Non-integer order integration by using neural networks, Proc. Int. Symp. Circuits Systems ISCAS'01, Vol. 3 (2001), pp. 688-691.

19. J. F. G. Aguilar, J. J. B. Alvarado, J. J. R. Garcia and T. C. Fraga, Modeling and simulation of equivalent circuits in description of biological systems - A fractional calculus approach, J. Electr. Bioimpedance 3 (2012) 2-11.

20. T. J. Freeborn, B. Maundy and A. S. Elwakil, Field programmable analogue array implementations of fractional step filters, IET Circuits Devices Syst. 4 (2010) 514-524.

21. I. Podlubny, I. Petras, B. Vinagre, P. O'Leary and L. Dorcak, Analogue realizations of fractional-order controllers, Nonlinear Dyn. 29 (2002) 281-296.

22. N. Sugimoto, Burgers equation with a fractional derivative; hereditary effects on nonlinear acoustic waves, J. Fluid Mech. 225 (1991) 631-653.

23. J. F. Gimez-Aguilar, J. J. Rosales-Garca, J. J. Bernal-Alvarado, T. Cardova-Fraga and R. Guzmn-Cabrera, Fractional mechanical oscillators, Rev. Mex. Fis. 58 (2012) $348-352$.

24. Y. Xu, Y. Lil and D. Liu, Responce of fractional oscillators with viscoelastic term under random excitation, J. Comput. Nonlinear Dyn. 9 (2013) 13-1213.

25. A. G. Radwan, A. M. Soliman and A . S. Elwakil, Design equations for fractional-order sinusoidal oscillators: Four practical circuits examples, Int. J. Circ. Theor. Appl. 36 (2007) 473-492.

26. W. Ahmad, R. El-Khazali and A. S. Elwakil, Fractional-order Wien-bridge oscillator, Electronics Lett. 37 (2001) 1110-1112.

27. A. G. Radwan, A. S. Elwakil and A. M. Soliman, Fractional-order sinusoidal oscillators: design procedure and practical examples, IEEE Trans. Circuits Syst. I Regul. Pap. $\mathbf{5 5}$ (2008) 2051-2063. 
28. A. S. Elwakil, A. Agambayev, A. Allagui and K. N. Salama, Experimental demonstration of fractional-order oscillators of orders 2.6 and 2.7, Chaos Solitons Fractals 96 (2017) $160-164$.

29. A. Kartci, N. Herencsar, J. Koton, L. Brančík, K. Vrba, G. Tsirimokou and C. Psychalinos, Fractional-order oscillator design using unity-gain voltage buffers and OTAs, Proc. 2017 60th IEEE Int. Midwest Symp. Circuits and Systems MWSCAS (Boston, USA, 2017) pp. 555-558.

30. Y. Shang, W. Fei and H. Yu, A fractional-order RLGC model for terahertz transmission line, IEEE MTT-S Int. Microw. Symp. Dig. (2013) 1-3.

31. C. Yang-Yang and S.-H. Yu, A compact fractional-order model for terahertz composite right/left handed transmission line, General Assembly and Scientific Symp. URSI GASS (Beijing, China, 2014), pp. 1-4.

32. R. Ismail and R. A. El-Barkouky, Fractional-order transmission line modeling, Int. J. Adv. Comput. Sci. Technol. 2 (2013) 18-24.

33. Z. Yanzhu and X. Dingyu, Dynamical simulation analysis based on time fractional transmission line model, 7th Int. Symp. Antennas, Propagation \& EM Theory, ISPE'06 (Guilin, China, 2006), pp. 1-4.

34. S. M. Cvetianin, D. Zorica and M. R. Rapaić, Generalized time-fractional telegraphers equation in transmission line modeling, Nonlinear Dyn. 88 (2017) 1453-1472.

35. X. Liu, X. Cui, L. Qi, G. Liang and L. Yan, Wide-band modeling of cables based on the fractional order differential theory, Proc. 2011 7th IEEE Asia-Pacific International Conf. Lightning APL (Chengdu, China, 2011), pp. 604-608.

36. E. C. Levy, Complex-curve fitting, IRE Trans. Autom. Control 1 (1959) 37-43.

37. A. Wardzinka and W. Badurski, Frequency-dependent and nonuniform parameters transmission line model, 20th IEEE Workshop Signal and Power Integrity SPI, Turin, Italy (2016), pp. 1-4.

38. L. Brančík, A. Kartci and N. A.-Z. R-Smith, Matlab simulation of transmission lines with skin effect via fractional telegraph equations and NILT, Proc. of 27th Int. Conf. European Association for Education in Electrical and Information Engineering EAEEIE, Grenoble, France (2017), pp. 1-5.

39. S. Lum, M. Nakhla and Q. J. Zhang, Sensitivity analysis of lossy coupled transmission lines with nonlinear terminations, IEEE Trans. Microwave Theory Tech. 39 (1991) 20892099.

40. N. A.-Z. R-Smith, A. Kartci and L. Brančík, Fractional-order lossy transmission line with skin effect using NILT method, Proc. 40th Int. Conf. Telecommunications and Signal Processing TSP (Barcelona, Spain, 2017), pp. 730-734.

41. L. Brančík, Numerical inverse Laplace transforms for electrical engineering simulation, MATLAB for Engineers - Applications in Control, Electrical Engineering, IT and Robotics, Edn. K. Perutka (InTech, Rijeka, 2011), pp. 51-74.

42. C. R. Paul, Analysis of Multiconductor Transmission Lines (John Wiley \& Sons, New York, 1994).

43. N. A.-Z. R-Smith, Accelerated hyperbolic NILT method used for frequency-dependent transmission line simulation, Proc. $23^{\text {rd }}$ Conf. Stud. EEICT (Brno, Czech Republic, 2017), pp. 634-639.

44. N. A.-Z. R-Smith and L. Brančík, Comparative study on one-dimensional numerical inverse Laplace transform methods for electrical engineering, Elektrorevue J. 18 (2016) $1-8$.

45. O. P. Agrawal, A general solution for a fourth-order fractional diffusion-wave equation defined in a bounded domain, Comput. Struct. 79 (2001) 1497-1501. 
46. A. Jalloul, K. Jelassi, P. Melchior and J. C. Trigeassou, Fractional identification of rotor skin effect in induction machines, Int. J. Comput. Sci. Issues 8 (2011) 57-68.

47. J. Machado and I. Jesus, Fractional order dynamics in some distributed parameter systems, Proc. 24th IASTED Int. Conf. Modeling, Identification and Control (Innsbruck, Austria, 2005), pp. 29-34.

48. S. Das, Functional Fractional Calculus (Springer-verlag, Berlin, 2008).

49. J. A. B. Faria, Multiconductor Transmission-Line Structures: Modal Analysis Techniques (John Wiley \& Sons, New York, 1993).

50. A. K. Goel, High-Speed VLSI Interconnections: Modeling, Analysis, and Simulation (John Wiley \& Sons, New York, 1994).

51. L. Brančík, Multiconductor transmission lines sensitivity via two-dimensional Laplace transform, Proc. 13th IEEE ICECS'2006, Nice, France (2006), pp. 17-20.

52. L. Brančík and B. Ševčík, Computer simulation of nonuniform MTLs via implicit Wendroff and state-variable methods, Radioengineering 20 (2011) 221-227.

53. L. Brančík, Improved method of numerical inversion of two-dimensional Laplace transforms for dynamical systems simulation, Proc. of 9th IEEE ICECS'2002, Dubrovnik, Croatia (2002), pp. 385-388.

54. L. Brančík, Simulation of multiconductor transmission lines using two-dimensional Laplace transformation, Proc. 15th European Conf. Circuit Theory and Design ECCTD'01, Espoo, Finland (2001), pp. 133-136.

55. E. C. Chang and S. M. Kang, Transient simulation of lossy coupled transmission lines using iterative linear least squares fitting and piecewise recursive convolution, IEEE Trans. Circuits Syst. I 43 (1996) 923-932.

56. L. Brančík, Utilization of quotient-difference algorithm in FFT-based numerical ILT method, Proc. of 11th Int. Czech-Slovak Scientific Conf. Radioelektronika'2001, Brno, Czech Republic (2001), pp. 352-355. 